\title{
Tunneling of conduction band electrons driven by a laser field in a double quantum dot: An open systems approach
}

\author{
B. Ahmadi, S. Salimi f $^{-}$and A. S. Khorashad \\ Department of Physics, Faculty of Science, \\ University of Kurdistan, Sanandaj, Iran
}

(Dated: April 17, 2018)

\begin{abstract}
In this paper, we investigate tunneling of conduction band electrons in a system of an asymmetric double quantum dot which interacts with an environment. First, we consider the case in which the system only interacts with the environment and demonstrate that as time goes to infinity they both reach an equilibrium, which is expected, and there is always a maximum and minimum for the populations of the states of the system. Then we investigate the case in which an external resonant optical pulse (a laser) is applied to the system interacting with the environment. However, in this case for different intensities we have different populations of the states in equilibrium and as the intensity of the laser gets stronger, the populations of the states in equilibrium approach the same constant.
\end{abstract}

PACS numbers:

\section{INTRODUCTION}

Developments in semiconductors have let us design quantum dots (QDs) [1 $\sqrt[3]{3}$. In these systems, few electrons may be confined in a nanometric space, which would be like atoms where few electrons are confined. A usual way to construct QDs is to trap a two-dimensional electronic gas in a semiconductor. This process of confinement creates a bowl-like potential in which conduction band electrons are trapped [4]. This is called a quantum dot. Quantum dots have let us make nanostructure devices which are governed by quantum mechanics rules [5 9]. It is predicted that in the future quantum dots will have numerous applications in industry like in memory chips [10], quantum computation [11, 12], and quantum cryptography [13]. In this work, we investigate a threelevel system made up of a double quantum dot. J. M. Villas-Boas et. al [14] have investigated such a system, but, what we do is totally different. In fact, here, a much more real system is considered, which interacts with the environment. The system is assumed to be a Markovian one [15]. The system and the bath can exchange energy, and this leads to dissipation, and fluctuations. Since the system is open and Markovian, the quantum optical master equation is used to investigate its evolution [15]. In the next section we let the system interact with the bath and write its equation of motion, then obtain and plot the results. In the third section we apply a laser to the system interacting with bath and then again write the appropriate equation of motion and plot the results.

\footnotetext{
*Electronic address: shsalimi@uok.ac.ir
}

†Electronic address: a.sorouri@uok.ac.ir

\section{SYSTEM INTERACTING WITH THE ENVIRONMENT}

The mutual influence of a system, with a few degrees of freedom, and a heat bath, with many degrees of freedom, on each other is the central concept in the physics of noise from both quantum and classical point of view. Here, the system under consideration is a QD-molecule formed from an asymmetric double QD system whose dots are coupled through tunneling and interacts with the bath. Such a system can be made by self-assembled dot growth technology [16]. The gap between energy levels of the right dot is much bigger than that of the left dot. Thus the energy levels are out of resonance, which means electron tunneling is weak (see Fig. 1(a)). But if we apply a voltage gate, the conduction band levels of the two dots get closer to resonance, which means their coupling increases, while the valence band levels become more off-resonant, meaning that their coupling becomes weak in a way that the probability of tunneling of the hole (left from the transition of the electron to the conduction band in the right dot) to the valence band of the right dot becomes very small (see Fig. 1(b)). Since the gap between the conduction band and the valence band is much bigger than that of the left dot we can assume that the bath is unable to excite the electrons of the valence band of the right dot (this is like a two-level atom interacting with the bath; although the system is a three-level system, the two of them are degenerate). It is also assumed that the system is initially in the state that there is no electron in the conduction band of the left dot (the state $|0\rangle$ ). Therefore when the system interacts with the bath, this interaction causes an electron to leave the valence band and jump into the conduction band and it leaves a hole in the valence band (the state $|1\rangle$ ) and then since the electron tunneling couples the conduction bands of the two dots, the electron can tunnel to the conduction band of the right dot, (the state $|2\rangle$ ).In this section 


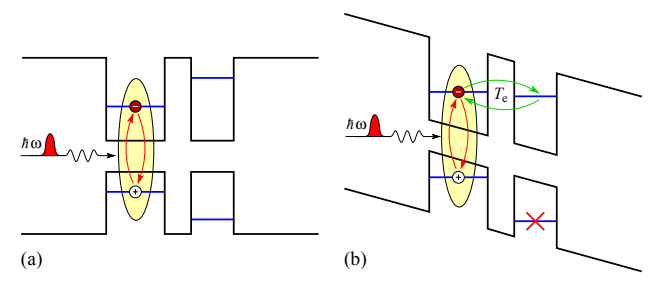

FIG. 1: (Color online) Schematic band structure of the system. (a) without a gate voltage. (b) with applied gate voltage.

we address the case in which the system only interacts with the bath and we see that the system and the bath reach an equilibrium as it is expected, but there would be some noticeable results about this equilibrium. The Hamiltonian of the total system can be written as

$$
H_{t o t}=H_{S}+H_{B}+H_{I}
$$

where $H_{S}$ is the Hamiltonian of the system and $H_{B}$ is the Hamiltonian of the bath and $H_{I}$ is the Hamiltonian describing the interaction between the system and the bath. The Hamiltonian of the uncoupled QD-molecule may be written in the form

$$
H_{S}=\sum_{i} \varepsilon_{i}|i\rangle\langle i|+T_{e}(|1\rangle\langle 2|+| 2\rangle\langle 1|),
$$

where $T_{e}$ is the electron tunneling matrix element which couples the conduction bands of the two dots. The heat bath which is taken to be a free quantized radiation field (with an infinite number of degrees of freedom) will be represented by the Hamiltonian (subtracting the zero point energy) [17],

$$
H_{B}=\sum_{j} \omega_{j} b_{j}^{\dagger} b_{j}
$$

where $b_{j}^{\dagger}$ and $b_{j}$ are the creation and annihilation operators of the field modes labeled by $\mathrm{j}$, respectively. In fact a field interpretation of the heat bath can almost always be possible. When viewed from the position of the system (like an atom or our QD-molecule here) there are two kinds of field modes, incoming and outgoing. The incoming modes affect the system, whereas the outgoing modes are produced by the system, they affect the system because they carry away energy, and thus give rise to damping. Incoming modes give the energy to the system, which is carried away by the outgoing modes. Thus the sort of damping produced is radiation damping. This view is true whenever a field interpretation of the bath is possible, and this is almost always possible [18. Finally, we assume the interaction Hamiltonian to be given in the dipole approximation by

$$
H_{I}=-\mathbf{D} . \mathbf{E},
$$

where $\mathbf{D}$ is the dipole operator of the system under consideration and $\mathbf{E}$ is the electric field operator in the
Schrödinger picture [15] which couples the states $|0\rangle$ and $|1\rangle$,

$$
\mathbf{E}=i \sum_{k} \sum_{\lambda=1,2} \sqrt{\frac{2 \pi \hbar \omega_{k}}{V}} \mathbf{e}_{\lambda}(k)\left(b_{\lambda}(k)-b_{\lambda}^{\dagger}(k)\right) .
$$

The system is completely similar to a three-level system, therefore we expect to have 6 Lindbland operators, two operators for each two levels [15]. But in our system the direct transitions $|0\rangle \rightleftarrows|2\rangle$ are neglected, as the dipole moment for that spatially indirect exciton will be vanishingly small. We thus have four Lindbland operators. For an arbitrary system interacting with the heat bath the quantum optical master equation in the Lindbland form would be 15.

$$
\begin{aligned}
\dot{\rho}(t) & =\sum_{\omega>0} \frac{4 \omega^{3}}{3 \hbar c^{3}}(N(\omega)+1)\left(\mathbf{A}(\omega) \rho(t) \mathbf{A}^{\dagger}(\omega)\right. \\
& \left.-\frac{1}{2}\left[\mathbf{A}^{\dagger}(\omega) \mathbf{A}(\omega), \rho(t)\right]_{+}\right) \\
& +\sum_{\omega>0} \frac{4 \omega^{3}}{3 \hbar c^{3}} N(\omega)\left(\mathbf{A}^{\dagger}(\omega) \rho(t) \mathbf{A}(\omega)\right. \\
& \left.-\frac{1}{2}\left[\mathbf{A}(\omega) \mathbf{A}^{\dagger}(\omega), \rho(t)\right]_{+}\right)-\frac{i}{\hbar}\left[H_{L S}, \rho_{S}(t)\right],
\end{aligned}
$$

where

$$
N(\omega)=\frac{1}{\exp (\beta \hbar \omega)-1}
$$

is the Planck distribution, which is the average number of photons in a mode with frequency $\omega$ and $\mathbf{A}(\omega)$ is called Lindbland operator and the last term is called the Lamb and Stark shift contribution. The terms $4 \omega^{3} / 3 \hbar c^{3}(N(\omega)+1)$ and $4 \omega^{3} / 3 \hbar c^{3} N(\omega)$ are the transition rates at which spontaneous and thermally induced emission and thermally induced absorption processes occur, respectively [15]. It should be mentioned that the quantum optical master equation is a Markovian master equation. Markovian behavior seems reasonable on physical grounds. Potentially, the system may depend on its past history. If, however, the bath is a large system maintained in thermal equilibrium, we do not expect it to preserve the minor changes brought by its interaction with the system for a very long time; not for long enough to significantly affect the future evolution of the system [19]. The interaction between matter and electromagnetic radiation is a typical and appropriate candidate for the application of quantum Markovian master equations [18, 20]. Lindbland operators are obtained by decomposing the dipole operator $\mathbf{D}$ into eigenvectors of $H_{S}$

$$
\mathbf{A}\left(\omega_{01}\right)=\langle 0|\mathbf{D}| 1\rangle|0\rangle\langle 1|,
$$

$$
\mathbf{A}^{\dagger}\left(\omega_{01}\right)=\langle 1|\mathbf{D}| 0\rangle|1\rangle\langle 0|,
$$




$$
\mathbf{A}\left(\omega_{12}\right)=\langle 1|\mathbf{D}| 2\rangle|1\rangle\langle 2|,
$$

$$
\mathbf{A}^{\dagger}\left(\omega_{12}\right)=\langle 2|\mathbf{D}| 1\rangle|1\rangle\langle 2| .
$$

Now we define

$$
\begin{aligned}
& \sigma_{-01}=|0\rangle\langle 1|, \\
& \sigma_{+01}=|1\rangle\langle 0|, \\
& \sigma_{-12}=|1\rangle\langle 2|, \\
& \sigma_{+12}=|2\rangle\langle 1|,
\end{aligned}
$$

and

$$
\begin{aligned}
& \mathbf{d}_{01}=\langle 0|\mathbf{D}| 1\rangle, \\
& \mathbf{d}_{01}^{*}=\langle 1|\mathbf{D}| 0\rangle, \\
& \mathbf{d}_{12}=\langle 1|\mathbf{D}| 2\rangle, \\
& \mathbf{d}_{12}^{*}=\langle 2|\mathbf{D}| 1\rangle .
\end{aligned}
$$

Therefore according to these definitions we have

$$
\begin{aligned}
& \mathbf{A}\left(\omega_{01}\right)=\mathbf{d}_{01} \sigma_{-01}, \\
& \mathbf{A}^{\dagger}\left(\omega_{01}\right)=\mathbf{d}_{01}^{*} \sigma_{+01}, \\
& \mathbf{A}\left(\omega_{12}\right)=\mathbf{d}_{12} \sigma_{-12}, \\
& \mathbf{A}^{\dagger}\left(\omega_{12}\right)=\mathbf{d}_{12}^{*} \sigma_{+12} .
\end{aligned}
$$

Substituting these Lindbland operators into Eq.(6) and neglecting the Lamb and Stark shift contribution [15], the quantum optical master equation can be written in the form

$$
\begin{aligned}
\dot{\rho}(t) & =\gamma_{01}\left(N\left(\omega_{01}\right)+1\right)\left(\sigma_{-01} \rho(t) \sigma_{+01}\right. \\
& \left.-\frac{1}{2}\left[\sigma_{+01} \sigma_{-01}, \rho(t)\right]_{+}\right) \\
& +\gamma_{01} N\left(\omega_{01}\right)\left(\sigma_{+01} \rho(t) \sigma_{-01}-\frac{1}{2}\left[\sigma_{-01} \sigma_{+01}, \rho(t)\right]_{+}\right) \\
& +\gamma_{12}\left(N\left(\omega_{12}\right)+1\right)\left(\sigma_{-12} \rho(t) \sigma_{+12}\right. \\
& \left.-\frac{1}{2}\left[\sigma_{+12} \sigma_{-12}, \rho(t)\right]_{+}\right)+\gamma_{12} N\left(\omega_{12}\right)\left(\sigma_{+12} \rho(t) \sigma_{-12}\right. \\
& \left.-\frac{1}{2}\left[\sigma_{-12} \sigma_{+12}, \rho(t)\right]_{+}\right),
\end{aligned}
$$

where

$$
\gamma_{i j}=\frac{4 \omega^{3}\left|\mathbf{d}_{i j}\right|^{2}}{3 \hbar c^{3}} .
$$

We want to investigate the case in which the levels $|1\rangle$ and $|2\rangle$ are in resonance. But the problem is that if we let $\omega_{12}$ be zero, the terms containing $\omega_{12}$ vanish, and this seems like we have a two-level system which is obviously not the case here. Thus to deal with this problem we do the following. Since $T_{e}$ is nonzero, therefore the transition rate from $|1\rangle$ to $|2\rangle$ must not vanish. Hence it stands to reason if we add a constant $\mathrm{n}$, proportional to $T_{e}$, to these transition rates. Therefore for the resonance case $\left(\omega_{12}=0\right)$, Eq.(8) reads

$$
\begin{aligned}
\dot{\rho}(t) & =\gamma_{01}\left(N\left(\omega_{01}\right)+1\right)\left(\sigma_{-01} \rho(t) \sigma_{+01}\right. \\
& \left.-\frac{1}{2}\left[\sigma_{+01} \sigma_{-01}, \rho(t)\right]_{+}\right) \\
& +\gamma_{01} N\left(\omega_{01}\right)\left(\sigma_{+01} \rho(t) \sigma_{-01}-\frac{1}{2}\left[\sigma_{-01} \sigma_{+01}, \rho(t)\right]_{+}\right) \\
& +n\left(\sigma_{-12} \rho(t) \sigma_{+12}-\frac{1}{2}\left[\sigma_{+12} \sigma_{-12}, \rho(t)\right]_{+}\right) \\
& +n\left(\sigma_{+12} \rho(t) \sigma_{-12}-\frac{1}{2}\left[\sigma_{-12} \sigma_{+12}, \rho(t)\right]_{+}\right) . \quad
\end{aligned}
$$

The matrix elements $\rho_{00}, \rho_{11}$ and $\rho_{22}$ are the populations of the states $|0\rangle,|1\rangle$ and $|2\rangle$, respectively. The offdiagonal elements $\rho_{i j}(i \neq j)$ are the coherences given by the expectation values of the raising and lowering operators $\sigma_{ \pm}$. The differential equations satisfied by $\rho_{00}$ and $\rho_{11}$ and $\rho_{22}$ are

$$
\begin{gathered}
\dot{\rho}_{00}(t)=l \rho_{11}(t)-m \rho_{00}(t), \\
\dot{\rho}_{11}(t)=-(l+n) \rho_{00}(t)+m \rho_{11}(t)+n \rho_{22}(t), \\
\dot{\rho}_{22}(t)=n \rho_{11}(t)-n \rho_{22}(t),
\end{gathered}
$$

where $l$ equals $\gamma_{01}\left(N\left(\omega_{01}\right)+1\right)$ and $m$ equals $\gamma_{01} N\left(\omega_{01}\right)$. Assuming that the system is initially in the state $|0\rangle$, one can solve these differential equations to obtain

$$
\begin{aligned}
\rho_{00}(t)= & \frac{l}{2 m+l}+B \exp \left(\lambda_{0} t\right)+C \exp \left(\lambda_{1} t\right), \\
\rho_{11}(t) & =\frac{m}{2 m+l}+\frac{\left(\lambda_{0}+m\right) B}{l} \exp \left(\lambda_{0} t\right) \\
& +\frac{\left(\lambda_{1}+m\right) C}{l} \exp \left(\lambda_{1} t\right), \\
\rho_{22}(t)= & \frac{m}{2 m+l}-\left(B+\frac{\left(\lambda_{0}+m\right) B}{l}\right) \exp \left(\lambda_{0} t\right) \\
- & \left(C+\frac{\left(\lambda_{1}+m\right) C}{l}\right) \exp \left(\lambda_{1} t\right),
\end{aligned}
$$


where $\mathrm{B}$ and $\mathrm{C}$ are constants and

$$
\begin{aligned}
& \lambda_{0}=\frac{-(l+m+2 n)-\sqrt{(l+m+2 n)^{2}-4(\ln +2 m n)}}{2}, \\
& \lambda_{1}=\frac{-(l+m+2 n)+\sqrt{(l+m+2 n)^{2}-4(\ln +2 m n)}}{2} .
\end{aligned}
$$

$\mathrm{B}$ and $\mathrm{C}$ can be determined from the initial condition. The terms including these constants vanish as time goes to infinity (See appendix (A)). It is clear that as time goes to infinity $\rho_{00}$ decreases exponentially to a constant which is $l /(2 m+l)$ and both $\rho_{11}$ and $\rho_{22}$ increase from zero to the same constant $m /(2 m+l)$. In other words, in the limit of $t \rightarrow \infty$ the solutions become stationary and this means that the system and the environment are in equilibrium. What is interesting is that the stationary values depend on $l$ and $m$ but not on $n$. $n$ just determines how fast $\rho_{22}(t)$ reaches its stationary value. The other interesting point is that the stationary value of $\rho_{11}$ and $\rho_{22}$ is always less than 0.33 in the absence of an external driving resonant optical pulse. In the next section, we investigate the above-mentioned total system in the presence of a laser and determine the condition under which $\rho_{11}$ and $\rho_{22}$ can reach this maximum. Since the transitions $|0\rangle \rightleftarrows|2\rangle$ are neglected, the system takes on the state $|1\rangle$ before the $|2\rangle$, thus $\rho_{11}$ reaches this constant faster than $\rho_{22}$ (see Figs. $2-4$ ).

\section{SYSTEM DRIVEN BY AN EXTERNAL RESONANT OPTICAL PULSE (A LASER)}

In this section we address the case in which the transition from $|0\rangle$ to $|1\rangle$ is driven by an external laser on resonance. The Hamiltonian in this case can be written as

$$
H_{t o t}=H_{S}+H_{B}+H_{I}+H_{L}
$$

where $H_{L}$ is the Hamiltonian of the external resonant optical pulse. Invoking the dipole approximation we obtain the following interaction picture Hamiltonian describing the interaction of the system with the driving mode:

$$
H_{L}(t)=-\mathbf{E}_{L}(t) \cdot \mathbf{D}(t),
$$

where

$$
\mathbf{E}_{L}(t)=\mathbf{e} \exp \left(-i \omega_{0} t\right)+\mathbf{e}^{*} \exp \left(i \omega_{0} t\right)
$$

is the electric field strength of the driving mode. Since the laser is on resonance with the transition from $|0\rangle$ to $|1\rangle$ therefore $\omega_{0}=\omega_{01}$. Now we can write $H_{L}(t)$ in the rotating wave approximation as follows,

$$
H_{L}=-\frac{\Omega}{2}\left(\sigma_{+01}+\sigma_{-01}\right)
$$

where the product

$$
\Omega=2 \mathbf{e} \cdot \mathbf{d}_{01}^{*}
$$

is refereed to as the Rabi frequency. Now neglecting the Lamb and Stark shift contribution again, the quantum optical master equation reads [15]

$$
\begin{aligned}
\dot{\rho}(t) & =-\frac{\Omega}{2}\left[\sigma_{+01}+\sigma_{-01}, \rho(t)\right]_{+} \\
& +\gamma_{01}\left(N\left(\omega_{01}\right)+1\right)\left(\sigma_{-01} \rho(t) \sigma_{+01}\right. \\
& \left.-\frac{1}{2}\left[\sigma_{+01} \sigma_{-01}, \rho(t)\right]_{+}\right) \\
& +\gamma_{01} N\left(\omega_{01}\right)\left(\sigma_{+01} \rho(t) \sigma_{-01}-\frac{1}{2}\left[\sigma_{-01} \sigma_{+01}, \rho(t)\right]_{+}\right) \\
& +n\left(\sigma_{-12} \rho(t) \sigma_{+12}-\frac{1}{2}\left[\sigma_{+12} \sigma_{-12}, \rho(t)\right]_{+}\right) \\
& +n\left(\sigma_{+12} \rho(t) \sigma_{-12}-\frac{1}{2}\left[\sigma_{-12} \sigma_{+12}, \rho(t)\right]_{+}\right) .
\end{aligned}
$$

Since for this case the elements of the density matrix are complex, we have 18 coupled differential equations. Diagonal elements are coupled with the off-diagonal ones. Numerically solving these equations we find that when time goes to infinity the results become stationary (see appendix A). But for different amounts of $2 l / \Omega$, relaxation times and the number of oscillations are different. The smaller $2 l / \Omega$ is, the more the number of oscillations and the longer relaxation time will be. This is clearly due to the fact that when the intensity of the laser increases, the laser drives more strongly the system to oscillate with the frequency $\omega$ which is the frequency of the laser. From now on we set $\Omega / 2$ equal to $p$ for simplicity. The results are plotted in figures $(5-13)$. We have not plotted $\rho_{22}(t)$ for $l / p=1, l / p=1 / 2$ and $l / p=1 / 10$ because its evolution is very similar to $\rho_{22}(t)$ when $l / p=2$ (i.e it exponentially increases from zero to a constant and the only difference is that these constants are different from each other for different values of $l / p$ and it approaches $1 / 3$ as the intensity of the laser increases.).

\section{CONCLUSION}

We investigated a system of a quantum dot molecule and saw that regardless of applying an external resonant optical pulse, as $t \rightarrow \infty$ the system and the environment always reaches an equilibrium, which is obvious and expected for a system interacting with an environment. In equilibrium the populations become stationary but as we see from Fig. 2 to Fig. 13 as $l / p$ decreases, a decrease in the stationary value of $\rho_{00}(t)$ begins and conversely an increase in those of $\rho_{11}(t)$ and $\rho_{22}(t)$. But the most important point is that as $l / p$ decreases the stationary values of $\rho_{00}(t), \rho_{11}(t)$ and $\rho_{22}(t)$ approach the same constant which is $1 / 3$. This means that the three populations become equal in equilibrium when $p \rightarrow \infty$. The reason is that the external laser causes the system to leave the 
state $|0\rangle$ and jump to the state $|1\rangle$, therefore the probability of finding the system in the state $|0\rangle$ decreases and accordingly the probability of finding the system in the state $|1\rangle$ and $|2\rangle$ increases. This in turn leads to a decrease in the population of the state $|0\rangle$ and consequently a decrease in the stationary value of $\rho_{00}(t)$ in equilibrium. This decrease continues as the intensity of the laser increases until the stationary value of $\rho_{00}(t)$ reaches 0.33 and then it stops decreasing, i.e the external laser causes the stationary value of $\rho_{00}(t)$ to encounter a decrease to the final value $1 / 3$. But the stationary values of $\rho_{11}(t)$ and $\rho_{22}(t)$ encounter an increase to the final stationary value $1 / 3$ as the laser is applied. In other words, as we increase the intensity of the laser, as a tunneling controlling parameter, from zero the stationary state of the system approaches a specific stationary state in which the probabilities of finding electron in the levels become equal and the maximum probability of tunneling from the conduction band of the left dot to the conduction band of the right dot becomes $1 / 3$. To sum up, in this work we showed that tunneling can be either increased or decreased depending on the intensity of the laser, but due to the presence of the environment there is always a maximum for the populations of the two conduction bands of both dots and a minimum for the population of the valence band of the left dot. The stronger the applied laser is the closer the population of the second dot will be to this maximum. We also saw whether the external driving mode is applied or not there is always an equilibrium which the system and the environment reach as $t \rightarrow \infty$.

\section{APPENDIX: A}

In the following we obtain the exact solutions of Eqs.(11), (12) and (13). Applying Laplace transform to these equations we have

$$
\begin{gathered}
s \rho_{00}(s)-\rho_{00}(t=0)=l \rho_{11}(s)-m \rho_{00}(s), \\
s \rho_{11}(s)-\rho_{11}(t=0)=-(l+n) \rho_{00}(s)+m \rho_{11}(s)+n \rho_{22}(s), \\
s \rho_{22}(s)-\rho_{22}(t=0)=n \rho_{11}(s)-n \rho_{22}(s), \\
\rho_{00}(s)+\rho_{11}(s)+\rho_{22}(s)=\frac{1}{s},
\end{gathered}
$$

where the last equality comes from the completeness relation, $\operatorname{tr} \rho=1$. After doing some calculations we find that

$$
\rho_{00}(s)=\frac{A}{s}+\frac{B}{s-\lambda_{0}}+\frac{C}{s-\lambda_{1}}
$$

where

$$
\lambda_{0}=\frac{-(l+m+2 n)-\sqrt{(l+m+2 n)^{2}-4(l n+2 m n)}}{2},
$$

$$
\lambda_{1}=\frac{-(l+m+2 n)+\sqrt{(l+m+2 n)^{2}-4(l n+2 m n)}}{2} .
$$

Now applying $\mathcal{L}^{-1}$ to $\rho_{00}(s)$ gives $\rho_{00}(t)$ as

$$
\rho_{00}(t)=A+B \exp \left(\lambda_{0} t\right)+C \exp \left(\lambda_{1} t\right)
$$

where $\mathrm{A}, \mathrm{B}$ and $\mathrm{C}$ are constants. $\mathrm{A}$ is the stationary value of $\rho_{00}(t)$ when time goes to infinity. Substituting $\rho_{00}(t)$ into Eqs.(11) and $\operatorname{tr} \rho=1$, one can easily obtain $\rho_{11}(t)$ and $\rho_{22}(t)$ as

$$
\begin{aligned}
\rho_{11}(t)= & \frac{m A}{l}+\frac{\left(\lambda_{0}+m\right) B}{l} \exp \left(\lambda_{0} t\right)+\frac{\left(\lambda_{1}+m\right) C}{l} \exp \left(\lambda_{1} t\right), \\
\rho_{22}(t) & =1-\left(A+\frac{m A}{l}\right)-\left(B+\frac{\left(\lambda_{0}+m\right) B}{l}\right) \exp \left(\lambda_{0} t\right) \\
& -\left(C+\frac{\left(\lambda_{1}+m\right) C}{l}\right) \exp \left(\lambda_{1} t\right) .
\end{aligned}
$$

The stationary values of $\rho_{00}(t), \rho_{11}(t)$ and $\rho_{22}(t)$ can be obtained by setting $\dot{\rho}_{00}(t), \dot{\rho}_{11}(t)$ and $\dot{\rho}_{22}(t)$ equal to zero. Therefore from Eq. (13) we have

$$
\rho_{11}(t)=\rho_{22}(t)
$$

Substituting Eqs.(33) and (34) into Eq.(35) one gets

$$
A=\frac{l}{2 m+l}
$$

which is the stationary value of $\rho_{00}(t)$. From the initial condition, $\rho_{00}(t=0)=1$ and $\rho_{11}(t=0)=0$, we find that

$$
B=1-\frac{l\left(m+(2 m+l)\left(\lambda_{0}-\lambda_{1}\right)\right)-2 m\left(\lambda_{0}+m\right)}{(2 m+l)\left(\lambda_{0}-\lambda_{1}\right)}
$$

and

$$
C=\frac{m\left(l+2\left(\lambda_{0}+m\right)\right)}{(2 m+l)\left(\lambda_{0}-\lambda_{1}\right)} .
$$

Substituting A into Eqs.(33) and (34) the stationary values of both $\rho_{11}(t)$ and $\rho_{22}(t)$ are obtained to be $m / 2 m+l$. Now consider the effect of the presence of an external resonant optical pulse in the stationary values of $\rho_{00}(t)$, $\rho_{11}(t)$ and $\rho_{22}(t)$. In this case there are 18 coupled complex equations but all the matrix elements of the density matrix vanish except real parts of $\rho_{00}(t), \rho_{11}(t)$ and $\rho_{22}(t)$ and imaginary parts of $\rho_{12}(t)$ and $\rho_{21}(t)$. Therefore we have five equations plus the completeness equation,

$$
\dot{\rho}_{00}(t)=l \rho_{11}(t)-m \rho_{00}(t)+p \rho_{21}(t)-p \rho_{12}(t),
$$

$$
\dot{\rho}_{11}(t)=-(l+n) \rho_{00}(t)+m \rho_{11}(t)+n \rho_{22}(t)-p \rho_{21}(t)+p \rho_{12}(t)
$$

$$
\dot{\rho}_{22}(t)=n \rho_{11}(t)-n \rho_{22}(t)
$$




$$
\begin{gathered}
\dot{\rho}_{12}(t)=-\frac{1}{2}(l+m+n) \rho_{12}(t)-p \rho_{11}(t)+p \rho_{00}(t), \\
\dot{\rho}_{21}(t)=-\frac{1}{2}(m+n) \rho_{12}(t)+p \rho_{11}(t)-p \rho_{00}(t), \\
\rho_{00}(t)+\rho_{11}(t)+\rho_{22}(t)=1 .
\end{gathered}
$$

Setting $\dot{\rho}_{00}(t), \dot{\rho}_{11}(t), \dot{\rho}_{22}(t), \dot{\rho}_{12}(t)$ and $\dot{\rho}_{21}(t)$ equal to zero, after some calculations, the stationary values of $\rho_{00}(t), \rho_{11}(t)$ and $\rho_{22}(t)$ can be easily obtained,

$$
\rho_{00}(t \rightarrow \infty)=\frac{l+2 p^{2}\left(\frac{1}{m+n}+\frac{1}{l+m+n}\right)}{2 l+m+6 p^{2}\left(\frac{1}{m+n}+\frac{1}{l+m+n}\right)},
$$

$\rho_{11}(t \rightarrow \infty)=\rho_{22}(t \rightarrow \infty)=1-\frac{2\left(l+2 p^{2}\left(\frac{1}{m+n}+\frac{1}{l+m+n}\right)\right)}{2 l+m+6 p^{2}\left(\frac{1}{m+n}+\frac{1}{l+m+n}\right)}$.

As can be seen from above equations when $p$ gets bigger, these stationary values approach $1 / 3$ and in the limit of $p \rightarrow \infty$ the three stationary values become $1 / 3$.
[1] S.N. Moltokov, S.S. Nazin, JETP Lett. 63 (1996) 687.

[2] H. Satio, K. Nishi, I. Ogura, s. Sogou, Y. Sugomito, Appl. Phys. Lett.69 (1996) 3140.

[3] P. Harison, Quantum Wells, Wires and Dots, Wiley, New York, 2006.

[4] T. Chakraborty, Quantum Dots, A survey of the properties of artificial atoms (Institute of Mathematical Sciences, Taramani, Madras 600113, India).

[5] D. Heitmann and J. P. Kotthaus, Physics Today, 46 (6), 56 (1993).

[6] M. A. Kastner, Physics Today, 46 (1), 24 (1993).

[7] W. Hansen, J. P. Kotthaus, and U. Merkt, Semiconductors and Semimetals, 32, 279 (1992).

[8] M. A. Reed, (Ed.), Nanostructured Systems (Academic, San Diego, 1992).

[9] M. A. Reed, Scientific American, 268, 98 (1993).

[10] T. J. Thornton, Pep. Prog. Phys. 58, 311 (1995).

[11] D. Loss and D. P. DiVincenzo, Phys. Rev. A 57, 120 (1998).

[12] S. Benjamin and N. F. Johnson, Appl. Phys. Lett. 70, 2321 (1997).

[13] C. S. Lent and P. D. Tougaw, J. Appl. Phys.74, 6227 (1993).

[14] arXiv: cond-mat/0403445v1.

[15] H.P. Breuer and F. Petruccione, The theory of open quantum systems (Oxford University Press, Oxford, 2002).

[16] P. M. Petroff, A. Lorke, and A. Imamoglu, Phys. Today, 54(5), 46 (2001).

[17] P. Lambropoulos, D. Petrosyan, Fundamentals of Quantum Optics and Quantum Information (Springer, 2007).

[18] C. W. Gardiner and P. Zoller, Quantum Noise (Springer, 2000).

[19] W. C. Schieve and J. W. Mddelton, International J. Quant. Chem., Quantum Chemistry Symposisum 11, 625 (1977).

[20] D. F. Walls and G. J. Milburn, Quantum Optics

\section{REFERENCES}

(Springer-Verlag, 1994).

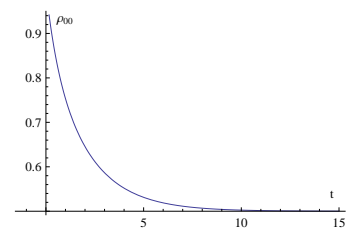

FIG. 2: $\rho_{00}(t)$ when $l=0.8, m=0.4$ and $\rho_{00}(t=0)=1$. As time goes to infinity $\rho_{00}(t)$ approaches a constant, $l /(2 m+l)$.

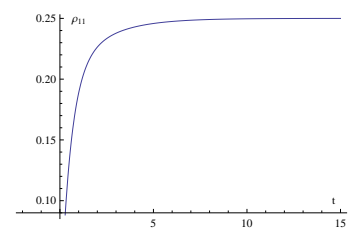

FIG. 3: $\rho_{11}(t)$ when $l=0.8, m=0.4$ and $\rho_{11}(t=0)=0$. As time goes to infinity $\rho_{11}(t)$ approaches a constant, $m /(2 m+l)$.

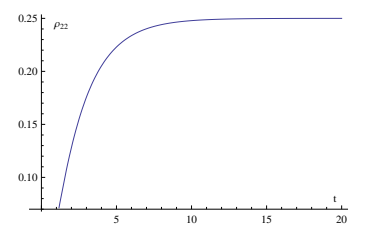

FIG. $4: \rho_{22}(t)$ when $l=0.8, m=0.4$ and $\rho_{22}(t=0)=0$. As time goes to infinity $\rho_{22}(t)$ approaches a constant, $m /(2 m+l)$. 


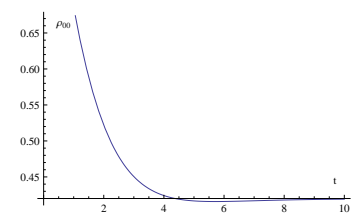

FIG. 5: $\rho_{00}(t)$ when $l / p=2, l=0.8, m=0.4$ and $\rho_{00}(t=$ $0)=1$. $\rho_{00}(t)$ has a slight oscillation. As time passes $\rho_{00}(t)$ approaches a constant.

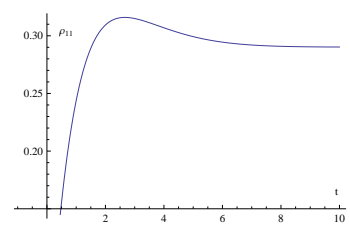

FIG. 6: $\rho_{11}(t)$ when $l / p=2, l=0.8, m=0.4$ and $\rho_{11}(t=$ $0)=0 . \rho_{11}(t)$ has a slight oscillation. As time passes $\rho_{11}(t)$ approaches a constant.

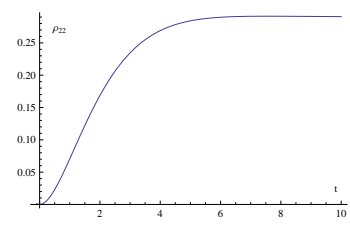

FIG. 7: $\rho_{22}(t)$ when $l / p=2, l=0.8, m=0.4$ and $\rho_{22}(t=$ $0)=0$. As time passes $\rho_{22}(t)$ increases and then approaches a constant.

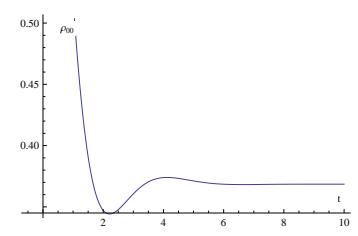

FIG. 8: $\rho_{00}(t)$ when $l / p=1, l=0.8, m=0.4$ and $\rho_{00}(t=$ $0)=1$. $\rho_{00}(t)$ begins to oscillate. As time passes the number of oscillations of $\rho_{00}(t)$ decreases and then $\rho_{00}(t)$ approaches a constant.

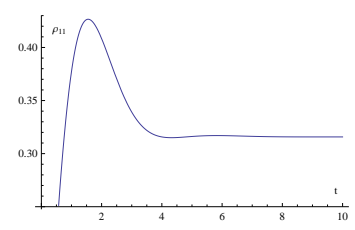

FIG. 9: $\rho_{11}(t)$ when $l / p=1, l=0.8, m=0.4$ and $\rho_{11}(t=$ $0)=0 . \rho_{11}(t)$ begins to oscillate. As time passes the number of oscillations of $\rho_{11}(t)$ decreases and then $\rho_{11}(t)$ approaches a constant. 


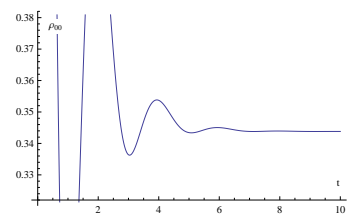

FIG. 10: $\rho_{00}(t)$ when $l / p=1 / 2, l=0.8, m=0.4$ and $\rho_{00}(t=$ $0)=1$. As time passes the number of oscillations of $\rho_{00}(t)$ decreases and then $\rho_{00}(t)$ approaches a constant.

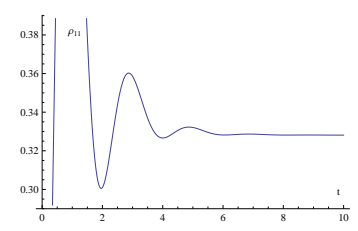

FIG. 11: $\rho_{11}(t)$ when $l / p=1 / 2, l=0.8, m=0.4$ and $\rho_{11}(t=$ $0)=0$. As time passes the number of oscillations of $\rho_{11}(t)$ decreases and then $\rho_{11}(t)$ approaches a constant.

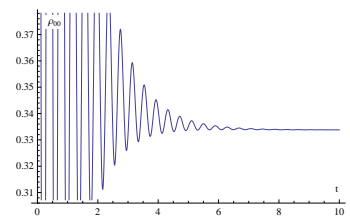

FIG. 12: $\rho_{00}(t)$ when $l / p=1 / 10, l=0.8, m=0.4$ and $\rho_{00}(t=0)=1$. As time passes the number of oscillations of $\rho_{00}(t)$ decreases and then $\rho_{00}(t)$ approaches a constant.

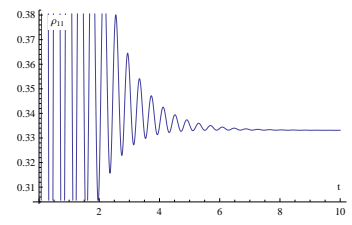

FIG. 13: $\rho_{11}(t)$ when $l / p=1 / 10, l=0.8, m=0.4$ and $\rho_{11}(t=0)=0$. As time passes the number of oscillations of $\rho_{11}(t)$ decreases and then $\rho_{11}(t)$ approaches a constant. 